\title{
A vibration method for measuring the thickness of concrete road slabs in situ*
}

\author{
by R. Jones, B.Sc., Ph.D.
}

Contribution by Professor J. W. H. King, M.Sc., M.I.C.E., M.I.Struct.E.

I was pleased to see at last some printed information on the latest brain child of Dr Jones. I had the pleasure of seeing this apparatus in action some months ago, and believe it can be a much more useful engineering tool than the ultrasonic pulse apparatus for which many exaggerated claims have been made in the recent past by wishful thinkers who did not study properly Dr Jones's paper on his apparatus and its use.

Given consistent concrete of uniform thickness, on a uniform base, this new technique should be easy to use, and judging by Table 1, quite reasonably accurate in its slab thickness assessment, using $V_{R}$ rather than $V_{L}$. It would appear probable also that $V_{R}$ might be at least as good as $V_{L}$ for assessment of concrete quality, in such a case-so rendering obsolescent the ultrasonic pulse velocity apparatus.

These are clearly quite considerable sources of error in practice. The slabs will vary in thickness, in compaction, and in actual concrete quality from point to point, so that with the need to use a distance of many wavelengths for any determination, there will be no precision about spot values. It is quite probable that often this will not be a disadvantage, since after all one must often assess quality on averages when site testing. It would be interesting to hear from Dr Jones if a bad patch on the line of test would give a characteristic non-linearity in the plots of Figure 3, from which the nature of its deficiencies could be assessed, and whether he found the causes of the anomalies in curves (c) and (d).

I believe Dr Jones has hopes of extending his findings into the region below the slab, and hope we may soon hear of his success in that direction.

\section{Contribution by G. Bradfield, B.Sc.†}

The method of measuring the thickness of concrete road slabs discussed by Dr Jones, using both low and high frequency vibrations launched at the free surface, must necessarily be somewhat inaccurate. There are four reasons for this. Firstly, a purely longitudinal wave does not propagate at grazing incidence to the surface of a

\footnotetext{
*Pages 97-102.

†Communicated from the National Physical Laboratory.
}

semi-infinite solid. $\left.{ }^{(1,}{ }^{2}\right)$ Some empirical correction (such as the $2 \%$ correction used by Dr Jones) must be employed and to what extent this correction is affected by local conditions is a matter for extensive experiment. Secondly, the surface of a concrete slab is somewhat different in constitution and hence in its elasticity from the lower layers (the lowest layers are, for quite other reasons, liable to still further deviations in elasticity). Thirdly, the use of continuous waves in Dr Jones's method involves the risk of reflections from slab lateral boundaries, e.g. from the normal road or road section edges or from faults in the form of cracks in the concrete; it seems unlikely, because of other difficulties with wave propagation, that the results of the measurements themselves can indicate what errors these reflections cause.

Finally, there is the influence of higher modes of propagation. The necessary limitation of the mode of excitation to the free surface causes both symmetrical and asymmetrical wave systems to propagate, each as principal wave and higher modes. It should be noted that Dr Jones's statement that, for the symmetrical wave system, the wave velocity approaches asymptotically to the Rayleigh wave velocity, is only true for the principal wave. In launching the asymmetrical wave system, the higher modes can be strong, ${ }^{(3)}$ indeed somewhat stronger than the principal wave. This may account for the discontinuities to be found in Figure 3 of the paper.

There are other complications connected with wave propagation in slabs, especially if Dr Jones's precaution of using " point" sources be disregarded: at wavelengths of the order of the slab thickness, abnormalities in the waves within a few wavelengths of the source may cause confusion in the observations.

The use of long wave trains in place of continuous waves may be helpful in avoiding errors due to the third point enumerated above. It remains to be seen whether extensive experiments will show that the method described is free from giving misleading results from the causes discussed above and it would be interesting to compare it with the method of echo sounding described by the writer and E. P. H. Woodroffe. ${ }^{(4)}$ With this method it appears possible to obtain both the thickness of the concrete and a value of $V_{L}=[E(1-\sigma) / \rho(1+\sigma)$ $(1-2 \sigma)]^{\frac{1}{2}}$ averaged approximately over the slab thickness. Further improvement may be expected using the techniques (e.g. of beam steering and short pulse generation) discussed by the writer ${ }^{(5)}$ and perhaps the methods of reduction of surface wave interference discussed by Pursey. ${ }^{(6)}$ 
An alternative echo method using shear waves generated by the torsional type of barium titanate transducer ${ }^{(7)}$ (this was shown at the British Instruments Industries Exhibition, 1955) may also be usable and could give additional information concerning the modulus of rigidity and the Poisson's ratio of the concrete.

\section{REFERENCES}

(1) MILLER, G. F. and PURSEY, H. The field and radiation impedance of mechanical radiators on the free surface of a semi-infinite isotropic solid. Proceedings of the Royal Society. Series A. Vol. 223. 1954. pp. 521-541.

(2) MILleR, G. F. and PURSEY, $\dot{H}$. On the partition of energy between elastic waves in a semi-infinite solid. Proceedings of the Royal Society. Series A. Vol. 233. 1955. pp. 55-69.

(3) PURSEY, $\mathrm{H}$. The launching and propagation of elastic waves in plates. (Awaiting publication.)

(4) BRADFIELD, G. and WOODROFFE, E. P. H. Determination of thickness of concrete pavement using mechanical waves. National Physical Laboratory Report No. Phys/U5. February 1953. pp. 9.

(3) BRADFIELD, G. Improvements in ultrasonic flaw detection. Journal of the British Institution of Radio Engineers. Vol. 14, No. 7. July 1954. pp. 303-308.

(6) PURSEY, H. The power radiated by an electro mechanical wave source. Proceedings of the Physical Society B. Vol. 69. 1956. pp. 139.

(7) Provisional Patent 3599/55. (Filed by the National Physical Laboratory and G. Bradfield.)

\section{Reply by the author}

I wish to thank Professor King and Mr Bradfield for their contributions, which both provide constructive ideas on the testing of concrete slabs. It is surprising to find, however, that their views seem somewhat at variance. Professor King welcomes the use of a method which provides an alternative to the ultrasonic pulse method, whereas $\mathrm{Mr}$ Bradfield is more critical of the sustained vibration method than of the pulse echo method.

Professor King, quite rightly, draws attention to the variations which may occur, in practice, in the quality and thickness of a concrete slab. 1 agree that an average assessment, as given by the sustained vibration method, may be of more value than a few spot results such as are given by echo methods. As yet, we have very little experience with other than experimental slabs containing concrete of reasonably consistent quality and, to the best of our knowledge, of uniform thickness. Variations in quality would certainly be shown up by corresponding variations in $V_{R}$ and, as Professor King suggests, we are using $V_{R}$ as a means of assessing the strength of the concrete. On all results to date $V_{R}$ has been extremely consistent on each slab, thereby indicating a uniform quality of concrete. The non-linearity of Figure 3 cannot, therefore, be attributed to variation in quality. In order to save any wishful thinking in the application of $V_{R}$ as a criterion of quality, it must be emphasized that the variables which affect the relation between pulse velocity and strength are also expected to affect the relation between $V_{R}$ and strength. Furthermore, as Mr Bradfield stresses, any measurement of velocity at the surface does not necessarily reflect the average quality of the concrete.

Professor King also refers to propagation at low frequencies for studying the underlying layers of the road. This work is very much in its infancy, although interesting results are being obtained, particularly on soil sites and flexible bituminous constructions.

Mr Bradfield draws attention to the inaccuracy of using a longitudinal wave at grazing incidence at the surface of the slab. This point appears to have been adequately stressed in my article and was one of the reasons for adopting a measurement of surface wave velocity $\left(V_{R}\right)$ rather than the pulse velocity $\left(V_{L}\right)$. I agree that the surface elastic properties of a slab may sometimes be different from the average elastic properties of the slab, though I would not agree with Mr Bradfield's implication that this is always so. However, as I have shown, any deterioration in quality with depth should be shown up as an equivalent reduction in thickness. I would agree with $\mathrm{Mr}$ Bradfield that measurements would be extremely difficult to make and to interpret on a badly cracked slab or near a discontinuity. So far, we have restricted our measurements to uncracked slabs and worked near the middle of the slab so that any reflections are considerably smaller than the direct vibrations.

I am very interested to hear that Mr Pursey has shown that the higher modes of propagation can sometimes be stronger than the principal mode, and I look forward to reading his forthcoming paper. By making measurements of amplitude variation in addition to change of phase, it may be possible to obtain more insight into the number and type of vibrations present. Our experience indicates that if, from results obtained at a number of frequencies, the smooth envelope of the lowest velocity at each frequency is drawn, this represents the principal mode of propagation. At very high frequencies the velocity we have obtained is in extremely good agreement with the Rayleigh wave velocity deduced from the dynamic elastic constants of the concrete, and we therefore presume that, in this range, we are also obtaining the principal mode of propagation.

Whilst it is appreciated that the sustained vibration method is not easy to apply or to interpret, we have had more success with it than with the echo method. The results obtained by $\mathrm{Mr}$ Bradfield and Mr Woodroffe by the echo method were from tests on small slabs with free surfaces and, even so, the accuracy of the estimates of $V_{L}$ and $T$ was rather poor $( \pm 10 \%)$. However, the improvements in technique which Mr Bradfield's research 
has evolved should help towards improving the accuracy of the echo methods. We have been hoping to put these improvements into practice in further tests by the echo method on an actual road slab, and to compare the echo method with the sustained vibration method, as Mr Bradfield suggests. It seems likely, however, that we may not be able to fit in these tests before the spring of 1956. 